\title{
Continuum and kinetic simulations of Heat Transfer trough Rarefied Gas in Annular and Planar Geometries in the Slip Regime
}

\author{
Mustafa Hadj-Nacer* \\ Research Scientist \\ Mechanical Engineering Department \\ University of Nevada, Reno \\ Reno, Nevada, 89557 \\ Email: mhadjnacer@unr.edu
}

\author{
Dilesh Maharjan \\ Graduate Research Assistant \\ Mechanical Engineering Department \\ University of Nevada, Reno \\ Reno, Nevada, 89557 \\ Email: dileshz@gmail.com
}

\author{
Minh-Tuan Ho \\ Research Associate \\ Department of Mechanical and Aerospace \\ University of Strathclyde \\ Glasgow, UK, G1 1XJ \\ Email:minh-tuan.ho@strath.ac.uk \\ Irina Graur \\ Professor \\ Mechanical Engineering Department \\ Aix-Marseille University \\ Marseille, France, 13453 \\ Email: irina.martin@univ-amu.fr
}

\section{Stefan K. Stefanov \\ Professor}

te of Mechanics

Bulgarian Academy of Science

Sofia, Bulgaria, 1113

Email: stefanov@imbm.bas.bg

\author{
Miles Greiner \\ Professor, ASME Fellow \\ Mechanical Engineering Department \\ University of Nevada, Reno \\ Reno, Nevada, 89557 \\ Email: greiner@unr.edu
}

\begin{abstract}
Steady-state heat transfer through a rarefied gas confined between parallel plates or coaxial cylinders, whose surfaces are maintained at different temperatures is investigated using the non-linear Shakhov (S) model kinetic equation and DSMC technique in the slip regime. The profiles of heat flux and temperature are reported for different values of gas rarefaction parameter $\delta$, ratios of hotter to cooler surface temperatures $\mathcal{T}$, and inner to outer radii ratio $\mathcal{R}$. The results of $S$-model kinetic equation and DSMC technique are compared to the numerical and analytical solutions of the Fourier equation subjected to the Lin \& Willis temperature-jump boundary condition. The analytical expressions are derived for temperature and heat flux for both geometries with hotter and colder surfaces having different values of the thermal accommodation coefficient. The results of the comparison between the kinetic and continuum approaches showed that the Lin \& Willis temperature-jump model accurately predicts heat flux and temperature profiles for small temperature ratio $\mathcal{T}=1.1$ and large radius ratios $\mathcal{R} \geq 0.5$, however, for large temperature ratio, a pronounced disagreement is observed.
\end{abstract}

\section{Introduction}

Used nuclear fuel assemblies consists primarily of square arrays of zircaloy cladding tubes containing highly-radioactive solids and gases [1]. They are initially stored underwater while their heat generation and radioactivity decrease [2]. After sufficient time individual assemblies are placed in square openings within helium-fill canisters. Before a canister is sealed, vacuum drying is used to remove remaining moisture [3]. During periods of time of this process the helium within the canister's narrow spaces is in the rarefied-gas slip regime. This causes the cladding temperature to increase compared to

\footnotetext{
*Address all correspondence to this author.
} 


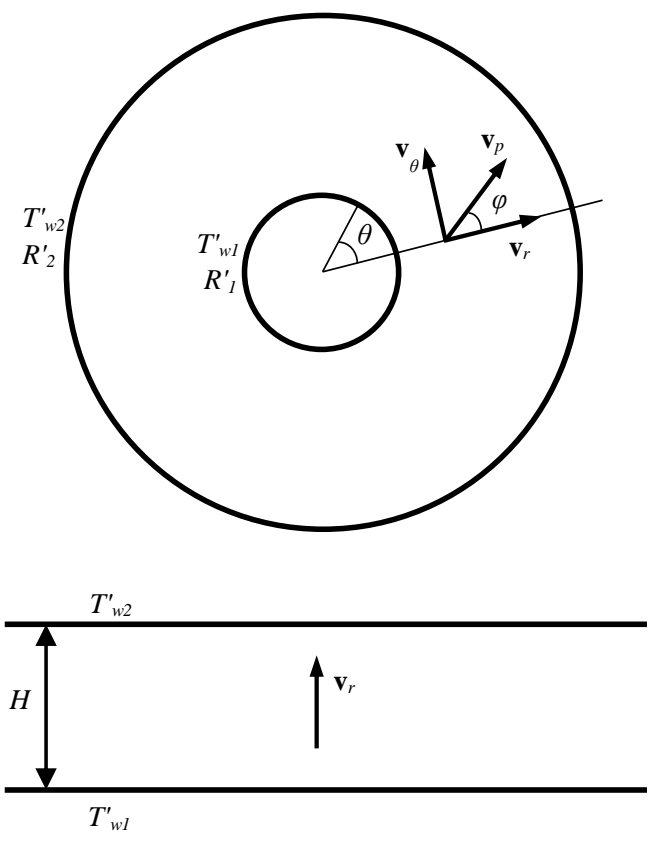

Fig. 1. Cross-section of (a) two coaxial cylinders and (b) two parallel plates configurations: dimensions $(r, \theta)$ in physical space, dimensions $\left(v_{r}, v_{\theta}\right)$ (or $\left.\left(v_{p}, \varphi\right)\right)$ in molecular velocity space.

continuum gas conditions. The cladding temperature must be accurately predicted to assure its temperature does not exceed certain important-to-safety limits [4].

The ultimate goal of this research is to develop and experimentally-benchmark computational methods to predict surface temperatures of complex void spaces containing slip-regime gases. For moderately low surface temperature differences and for surfaces with radii of curvature that are larger than the mean free path (mean distance traveled by molecules between two successive collisions), transport across slip-regime gases may be approximated using continuum Fourier conduction in the gas bulk, and temperature-jumps boundary condition at its interfaces with heated surfaces [5].

In the current paper, conduction across annular gaps filled with slip regime helium calculated from two kinetic gas methods (Shakhov-model [6,7,8] and Direct Simulation Monte Carlo [9]) are compared to calculations from continuum models with temperature-jump boundary conditions. Annular gaps with a range of radius ratios are simplified models for regions within used fuel canister and other engineering systems. In this paper, comparisons are made between the continuum and kinetic models for a range of radius ratios, surface temperature ratios, gas rarefaction parameters and surface accommodation coefficients. These comparisons are used to determine the conditions where the continuum models with temperature-jump boundary conditions may be used with confidence for the simple annular gap geometry. Future work will compare continuum and kinetic models for calculating heat transfer within an enclosed array of heated rods with experiment data in order to determine when the continuum models may be used in complex configurations.

\section{Problem formulation}

The problem of conduction heat transfer between parallel plates and coaxial cylinders separated a gas at rest is considered here (see Fig. 1). The radii and temperatures of the inner and outer cylinders are $\left(R_{1}^{\prime}, T_{w_{1}}^{\prime}\right)$ and $\left(R_{2}^{\prime}, T_{w_{2}}^{\prime}\right)$, respectively, with $R_{1}^{\prime}<R_{2}^{\prime}$ and $T_{w_{1}}^{\prime}>T_{w_{2}}^{\prime}$. For simplicity, the same notation is used for parallel plates: the $r^{\prime}$ axis is normal to both plate's surfaces. The bottom and upper plates have the locations and temperatures $\left(R_{1}^{\prime}, T_{w_{1}}^{\prime}\right)$ and $\left(R_{2}^{\prime}, T_{w_{2}}^{\prime}\right)$, respectively, with $R_{2}^{\prime}=-R_{1}^{\prime}=H^{\prime} / 2$. The cylinders and plates are assumed to have infinite length in the direction perpendicular to the figure.

Both problems are similar, however, the coaxial cylinder problem is governed by three physical parameters (temperature and radius ratios, and rarefaction parameter; see definitions below). The parallel plate problem is defined by only two parameters (temperature ratio and rarefaction parameter). It is convenient to introduce the following parameters:

- The temperature ratio between the cylinders or plates: $\mathcal{T}=T_{w_{1}}^{\prime} / T_{w_{2}}^{\prime}$,

- The aspect ratio for the cylinders and plates: $\mathcal{R}=\left|R_{1}^{\prime} / R_{2}^{\prime}\right|$, 
- The rarefaction parameter

$$
\delta=\frac{R_{0}}{\ell}, \quad \text { where } \quad \ell=\frac{\mu_{0} v_{0}}{p_{0}}, \quad v_{0}=\sqrt{\frac{2 k_{B} T_{0}}{m}}
$$

In these expressions, $\ell$ is the equivalent mean free path at reference pressure $p_{0}, m$ is the molecular mass of the gas, $k_{B}$ is the Boltzmann constant, and $v_{0}$ is the most probable molecular velocity at reference temperature $T_{0}$.

In expression (1), $R_{0}$ and $T_{0}$ are the reference space dimension and temperature, respectively, and $\mu_{0}$ is the gas viscosity calculated at the reference temperature; $\mu_{0}=\mu\left(T_{0}\right)$.

It is convenient to take the distance between the cylinders (or plates) as the reference space dimension $R_{0}=R_{2}^{\prime}-R_{1}^{\prime}$. The temperature of the external cylinder (or upper plate) is used as the reference temperature, $T_{0}=T_{w_{2}}^{\prime}$. The problem of heat transfer considered here is one dimensional, therefore, the heat flux has only one component in the direction normal to the surfaces, denoted by $q^{\prime}$.

For practical reasons, the following dimensionless variables are introduced

$$
r=\frac{r^{\prime}}{R_{0}}, \quad p=\frac{p^{\prime}}{p_{0}}, \quad T=\frac{T^{\prime}}{T_{0}} \quad \text { and } \quad q=\frac{q^{\prime}}{p_{0} v_{0}} .
$$

The influence of the gas-surface interaction is taken into account by the thermal accommodation coefficient, denoted as

$$
\alpha=\frac{T_{i}-T_{r}}{T_{i}-T_{w}},
$$

where $T_{i}$ and $T_{r}$ are the temperature of the incident and reflected molecules, respectively.

\section{Continuum approach}

The analytical expressions for heat flux and temperature can be obtained from an energy balance. The hypothesis of zero macroscopic velocity and constant pressure between the plates or cylinders are used to derive the analytical expressions. The Fourier law can be applied to calculate the heat flux

$$
q^{\prime}=-\kappa^{\prime} \frac{d T^{\prime}}{d r^{\prime}}
$$

For monatomic gases, the gas thermal conductivity $\kappa^{\prime}$ is related to the gas viscosity $\mu^{\prime}$ as follows [9]

$$
\kappa^{\prime}=\frac{15}{4} \frac{k_{B}}{m} \mu^{\prime}
$$

In order to define the dependence of viscosity on temperature, the molecular interaction potential must be specified. In this work, the inverse power law potential [9] is employed, which leads to a power law temperature dependence for the viscosity,

$$
\mu^{\prime}=\mu_{0}\left(\frac{T^{\prime}}{T_{0}}\right)^{\omega}
$$

where $\omega$ is the viscosity index, which is equal to 0.5 for the Hard-Sphere (HS) model and 1 for the Maxwell model. Its value varies as a function of the gas nature in the case of Variable Hard-Sphere (VHS) model, see [9]. In this paper, the Hard-Sphere model is retained for all calculations, so $\omega=0.5$ for all gases.

In the continuum regime, the temperature continuity condition may be assumed on the surfaces. However, in the slip regime, the temperature-jump conditions [10] must be used as boundary conditions,

$$
T_{g}^{\prime}=T_{w}^{\prime}+\left.\xi_{T} \ell \frac{d T^{\prime}}{d r^{\prime}}\right|_{w},
$$


where $T_{g}^{\prime}$ is the gas temperature near the wall and $\xi_{T}$ is the temperature-jump coefficient [11]. The dimensionless form of the temperature-jump boundary condition at the cylinders and plates walls can be written as

$$
T= \begin{cases}T_{w 1}+\frac{\xi_{T 1}}{\delta} T_{w 1}^{\omega+1 / 2} \frac{d T}{d r}, & r=R_{1} \\ T_{w 2}-\frac{\xi_{T 2}}{\delta} T_{w 2}^{\omega+1 / 2} \frac{d T}{d r}, & r=R_{2}\end{cases}
$$

Here $\xi_{T 1}, \xi_{T 2}$ are the temperature-jump coefficients of the hotter and colder surfaces, respectively. The assumption of the constant pressure between the cylinders and plates is used to obtain the previous expressions. The coefficient $\xi_{T}$ in Eq. (7) and (8) depends on the gas nature and surface state through the thermal accommodation coefficient. Lin and Willis, 1972 [12] proposed the following expression for polyatomic gases,

$$
\xi_{T}=\left(\frac{2-\alpha}{\alpha}+0.17\right) \frac{\sqrt{\pi}}{\operatorname{Pr}} \frac{\gamma}{\gamma+1}
$$

obtained by applying a variational method to the Morse equation [13] and to the Holway model [14]. In Eq. $9 \gamma$ is the gas specific heat ratio and $\mathrm{Pr}$ is the Prandtl number. For monatomic gases, this expression is reduced to the one proposed by Welander [15]. For the case of complete accommodation $(\alpha=1)$ and a monatomic gas $(\gamma=5 / 3$ and $\operatorname{Pr}=2 / 3)$, the value of this coefficient is $\xi_{T} \sim 1.95$.

In this paper, Eq (4) subjected to the boundary condition (8) is solved numerically using an iterative method for parallel plates and coaxial cylinders having different temperature and aspect ratios at different values of the rarefaction parameter $\delta$ in the slip regime, and various values of the thermal accommodation coefficient $\alpha$.

\subsection{Analytical solution}

To obtain the completely explicit expression of the temperature distribution between the cylinder and plates, a linearization of the temperature is carried out and the terms of the order of $\varepsilon^{2}$ are neglected, where $\varepsilon=\frac{T_{w}-T_{g}}{T_{w}}$ (Eq. 7), so the temperature profile between the cylinders and plates is obtained analytically with accuracy of $\varepsilon^{2}$.

\subsubsection{Parallel plates}

The following dimensionless analytical expressions are obtained for the temperature profile

$$
\begin{aligned}
T(r)=\left\{\frac{1}{2}[\right. & \left(T_{g 1}^{\omega+1}+T_{g 2}^{\omega+1}\right) \\
& \left.\left.+C(\omega+1)\left(R_{1}+R_{2}-2 r\right)\right]\right\}^{\frac{1}{\omega+1}}
\end{aligned}
$$

and heat flux profile

$$
q(r)=\frac{15}{8 \delta} C
$$

where

$$
C=\frac{\left(T_{w 1}^{\omega+1}-T_{w 2}^{\omega+1}\right) /(\omega+1)}{R_{2}-R_{1}+\frac{\xi_{T 1}}{\delta} T_{w 1}^{\omega+1 / 2}+\frac{\xi_{T 2}}{\delta} T_{w 2}^{\omega+1 / 2}} .
$$

The gas temperatures near the walls are

$$
\begin{aligned}
T_{g 1}^{\omega+1} & =T_{w 1}^{\omega+1}\left[1-\eta_{1}(\omega+1)\right], \\
T_{g 2}^{\omega+1} & =T_{w 2}^{\omega+1}\left[1-\eta_{2}(\omega+1)\right],
\end{aligned}
$$


where

$$
\eta_{1}=\frac{\xi_{T 1}}{\delta} C \frac{1}{T_{w 1}^{1 / 2}}, \quad \eta_{2}=-\frac{\xi_{T 2}}{\delta} C \frac{1}{T_{w 2}^{1 / 2}}
$$

Here, the dimensionless coordinates $R_{1}$ and $R_{2}$ for parallel plates are: $R_{1}=-0.5$ and $R_{2}=0.5$.

\subsubsection{Coaxial cylinders}

By implementing the same technique, the temperature and heat flux profiles for coaxial cylinders were obtained, as in Ref. [16]. The dimensionless temperature profile with an accuracy of $\varepsilon^{2}$ is

$$
\begin{aligned}
T(r) & =\left\{\frac { 1 } { 2 } \left[\left(T_{g 1}^{\omega+1}+T_{g 2}^{\omega+1}\right)\right.\right. \\
& \left.\left.+C(\omega+1)\left(\ln \left(\frac{R_{1}}{r}\right)+\ln \left(\frac{R_{2}}{r}\right)\right)\right]\right\}^{\frac{1}{\omega+1}},
\end{aligned}
$$

and the dimensionless heat flux profile is

$$
q(r)=\frac{15}{8 \delta} \frac{C}{r}
$$

where

$$
C=\frac{\left(T_{w 1}^{\omega+1}-T_{w 2}^{\omega+1}\right) /(\omega+1)}{\ln \frac{R_{2}}{R_{1}}+\frac{\xi_{T 1}}{\delta} \frac{T_{w 1}^{\omega+1 / 2}}{R_{1}}+\frac{\xi_{T 2}}{\delta} \frac{T_{w 2}^{\omega+1 / 2}}{R_{2}}} .
$$

In this paper, two aspect ratios are considered, $R=0.5$ and $R=0.1$. The corresponding dimensionless coordinate $\left[R_{1}\right.$, $\left.R_{2}\right]$ for each case are: $[1,2]$ and $[0.11,1.11]$, respectively. The gas temperature in the vicinity of the walls is calculated with the same expressions (13) as for the two parallel plates, with the parameters $\varepsilon_{1}$ and $\varepsilon_{2}$ calculated as

$$
\eta_{1}=\frac{\xi_{T 1}}{\delta} \frac{C}{R_{1}} \frac{1}{T_{w 1}^{1 / 2}}, \quad \eta_{2}=-\frac{\xi_{T 2}}{\delta} \frac{C}{R_{2}} \frac{1}{T_{w 2}^{1 / 2}}
$$

The analytical model is more convenient to calculate the temperature profile between the cylinders and plates than the numerical model as it needs no iteration.

\section{Kinetic approach}

Two kinetic models are used in this paper to simulate heat transfer between parallel plates and coaxial cylinders in the slip regime.

\subsection{S-model kinetic equation}

In the steady-state case the S-model kinetic equation [6] reads

$$
\mathbf{v} \frac{\partial f^{\prime}}{\partial \mathbf{r}^{\prime}}=\mathbf{v}^{\prime}\left(f^{s^{\prime}}-f^{\prime}\right)
$$

where, $f^{\prime}\left(\mathbf{r}^{\prime}, \mathbf{v}\right)$ is the one particle molecular velocity distribution function, $\mathbf{v}$ is the molecular velocity vector, $\mathbf{r}^{\prime}$ is the position vector, and $v^{\prime}$ is the molecular collision frequency. It should be underlined that Eq.(19) is valid if a Cartesian frame is used in the velocity space. In the case of the two coaxial cylinders, an axisymmetric problem, the transport part of Eq.(19) can be written in form used in Refs. [17], [8]. 
The equilibrium distribution function $f^{S^{\prime}}$ is

$$
\begin{aligned}
f^{S^{\prime}} & =f^{M^{\prime}}\left[1+\frac{2 m \mathbf{V} \mathbf{q}^{\prime}}{15 n^{\prime}\left(k_{B} T^{\prime}\right)^{2}}\left(\frac{m \mathbf{V}^{2}}{2 k_{B} T^{\prime}}-\frac{5}{2}\right)\right], \\
f^{M^{\prime}}\left(n^{\prime}, T^{\prime}\right) & =n^{\prime}\left(\frac{m}{2 \pi k_{B} T^{\prime}}\right)^{3 / 2} \exp \left[-\frac{m\left(\mathbf{v}-\mathbf{u}^{\prime}\right)^{2}}{2 k_{B} T^{\prime}}\right],
\end{aligned}
$$

where $f^{M^{\prime}}$ is the local Maxwellian distribution function, $\mathbf{u}^{\prime}$ is the bulk velocity vector, $\mathbf{V}=\mathbf{v}-\mathbf{u}^{\prime}$ is the peculiar velocity vector, and $\mathbf{q}^{\prime}$ is the heat flux vector.

In the frame of this model, the molecular collision frequency is assumed to be independent of the molecular velocities and may be found as follows [6]

$$
v^{\prime}=\frac{p^{\prime}}{\mu^{\prime}} .
$$

The macroscopic parameters are calculated from the solution of the molecular distribution function $f^{\prime}$ obtained by solving eq. (19), as

$$
\begin{aligned}
T^{\prime}\left(r^{\prime}\right) & =\frac{m}{3 n^{\prime} k_{B}} \iint \mathbf{V}^{2} f^{\prime}\left(\mathbf{r}^{\prime}, \mathbf{v}\right) d \mathbf{v} \\
q^{\prime}\left(r^{\prime}\right) & =\frac{m}{2} \iint \mathbf{V} V^{2} f^{\prime}\left(\mathbf{r}^{\prime}, \mathbf{v}\right) d \mathbf{v} .
\end{aligned}
$$

Considering the axial symmetry of the problem, the S-model kinetic equation in the completely conservative form is found in Refs. [8], [18], and [19]. The generalization of a kinetic model for the case of the polyatomic gases is presented in [20].

The Discrete Velocity Method (DVM) is used to divide the continuum-molecular velocity space $c_{p}$ in the system of kinetic equations (see Ref. [8]) into a discrete velocity set $c_{p_{k}}$. The system of kinetic equations with discrete velocity set $c_{p_{k}}$ is then discretized in space by the Finite Difference Method (FDM). The spatial derivatives are approximated by the second order-of-accuracy upwind-type numerical scheme. The Gauss-Hermite quadrature formulas are used to evaluate the integrals for calculating the macroscopic parameters (Eq. 22).

In the physical space, the reduced distribution functions depend only on the spatial variable $r$, which is either the distance from the cylinder's axis or the distance from midway between the plates. For coaxial cylinders, the distance between the walls is split into $N_{r}$ intervals, $N_{r}=800$ for $\delta \leq 10$ and $N_{r}=6400$ for $\delta>10$. For parallel plates, $N_{r}=4000$ for all $\delta$. In the velocity space, the distribution functions depend on two variables; the magnitude and orientation of the molecular velocity vector $c_{p_{k}}$ and $\varphi$, respectively. The velocity vector magnitude $c_{p_{k}}$ is distributed according to the Gaussian quadrature rule, which is characterized by Gaussian abscissas $N_{c_{p}}$ and their corresponding weights. The number of implemented points $N_{c_{p}}$ depends on gas rarefaction; $N_{c_{p}}=25$ for $\delta<0.5$ and $N_{c_{p}}=12$ for $\delta \leq 0.5$. The range of molecular velocity orientations $(0 \leq \varphi \leq \pi)$ is divided into $N_{\varphi}$ equal intervals. For coaxial cylinders, $N_{\varphi}=6400$ for $\delta \leq 10$ and $N_{\varphi}=100$ for larger $\delta$. For parallel plates, $N_{\varphi}=100$ for all $\delta$. Moreover, this range of $\varphi$ is divided into two sub-domains, according to the sign of the molecular velocity components. This set of the numerical grid parameters guarantees the accuracy for energy conservation law of the order of $0.1 \%$.

\subsection{Direct Simulation Monte Carlo (DSMC)}

The traditional DSMC technique proposed by Bird [9] is employed in this paper for the calculation of heat transfer between parallel plates and coaxial cylinders. The DSMC technique enables gas flows to be modeled on a molecular level by simulating the motion of individual particles according to their physical properties. This technique can be viewed as a Monte Carlo method for solving the time-dependent non-linear Boltzmann equation. Within each time step $t$ of the simulation, this method combines deterministic aspects for modeling particle motions with statistical aspects for computing collisions between particles. The collision technique that is used is the NoTime Counter scheme suggested by Bird, with a slight modification in the calculation of maximum number of collision in a cell, as described in Ref. [21].

The procedure for axis-symmetric flows is employed as given in [9]. For each particle, only the radial coordinate $r$ and the three velocity components $\mathbf{v}_{\mathbf{x}}, \mathbf{v}_{\mathbf{r}}$, and $\mathbf{v}_{\theta}$ are stored. Then, the molecules are moved according to their velocities and 
acquire a new radial coordinate $r^{+}$given by

$$
r^{+}=\sqrt{\left(r+\mathbf{v}_{\mathbf{r}} \Delta t\right)^{2}+\left(\mathbf{v}_{\theta} \Delta t\right)^{2}}
$$

Following this procedure, the new velocity components, $\mathbf{v}_{\mathbf{x}}^{+}, \mathbf{v}_{\mathbf{r}}^{+}$, and $\mathbf{v}_{\mathbf{z}}^{+}$, are

$$
\begin{aligned}
& \mathbf{v}_{\mathbf{x}}^{+}=\mathbf{v}_{\mathbf{x}}, \\
& \mathbf{v}_{\mathbf{r}}^{+}=\frac{\mathbf{v}_{\mathbf{r}}\left(r+\mathbf{v}_{\mathbf{r}} \Delta t\right)-\mathbf{v}_{\theta}^{2} \Delta t}{r^{+}}, \quad \text { and } \\
& \mathbf{v}_{\mathbf{z}}^{+}=\frac{\mathbf{v}_{\mathbf{r}} \mathbf{v}_{\theta} \Delta t-\mathbf{v}_{\theta}\left(r+\mathbf{v}_{\mathbf{r}} \Delta t\right)}{r^{+}} .
\end{aligned}
$$

The domain is discretized in the radial direction using $N_{r}$ computational cells ( $N_{r}$ ranges from 400 to 4000$)$ and the particles are initially distributed in such a way that the minimum number of particles in each cell of the domain is $N_{p}=50$. Since no weighting factors are used, the region where the number of particles per cell is the smallest corresponds to the area near the inner cylinder for geometrical reasons. The time step $\Delta t$ is chosen to be less or equal to one third the cell traversal time, $\Delta r / \sqrt{2 k T_{0} / m}$. The macroscopic properties are obtained for the steady state solution by time averaging over $N_{T}=10000$ time steps. The heat flux and other macroscopic quantities are volume based calculated by averaging the microscopic values of the particles at a given cell, $q_{r}=\overline{\mathbf{v}_{\mathbf{r}} \mathbf{v}^{2}}$. For these grid and time parameters, the total computational error (systematic and statistical) is less than $1 \%$ in all the computed cases. Here, the available analyses of the splitting scheme and domain discretization [22,23] suggest a first order systematic approximation error of $(O(\Delta t, \Delta r))$, with respect to the Boltzmann equation. The statistical error [24] is estimated as $O\left(1 / \sqrt{N_{T} N_{p}}\right)$.

It should be noted here that the computer code described above was employed for two coaxial cylinders with $\mathcal{R}=0.1$ and 0.5 , and two parallel plates with $\mathcal{R}=0.999$.

\section{Results and Discussions}

In this section, results of steady-state heat transfer, between parallel plates and coaxial cylinders in the slip regime, obtained from kinetic and continuum approaches, are presented. The results are showed in graphical form for the macroscopic quantities (temperature, heat flux, and pressure) in term of all the quantities involved in the problem. For all three geometries, parallel plates $(\mathcal{R}=1)$ and coaxial cylinders $(\mathcal{R}=0.5$ and 0.1$)$, two temperature ratios $\mathcal{T}=1.1$ and 2 are examined. The value of the rarefaction parameter $\delta$ is varied from 500 to 3 , which covers the continuum, slip, and near transitional regimes [25]. In this work, we wish to determine the conditions where the difference between the continuum and kinetic results of the same order as the difference between the S-model and DSMC results.

\subsection{Dimensionless temperature profiles}

In order to assess the limit of applicability of the continuum models, first, a comparison of the dimensionless temperature profiles obtained from the S-model and DSMC technique is performed. Figure 2 shows the dimensionless temperature distribution along the $r$-axis, obtained from the kinetic (S-model and DSMC) approaches and the continuum (numerical and analytical) models for the temperature and radius ratios considered in this work, in the case of full accommodation of the molecules at the walls $\left(\alpha_{1}=\alpha_{2}=1\right)$ and for different values of rarefaction parameter $\delta=100,50,10$ and 3 .

Comparison between the profiles of dimensionless temperature obtained from the kinetic model shows that the S-model and DSMC technique are in good agreement for all $\mathcal{T}, \mathcal{R}$, and $\delta$ employed in this paper. Both models exhibit the same profile even in the Knudsen layers (local non-equilibrium region with a thickness $\sim O(\ell)$ from the wall).

As expected, for $\mathcal{T}=1.1$, the profiles of dimensionless temperature from the continuum numerical and analytical (10, 15) models exhibit a nearly linear trend for parallel plates and a nearly logarithmic trend for coaxial cylinders. This is due to the relatively small thermal conductivity variation across the gaps. For the smaller temperature ratio $(\mathcal{T}=1.1$, left plots), the numerical and analytical temperature profiles are very similar for all values of $\delta$, but the difference increases as $\mathcal{R}$ decreases. For the larger temperature ratio ( $\mathcal{T}=2$, right plots), the difference between the two continuum models is larger and increases as $\delta$ decreases. In all cases, the temperatures predicted by the numerical model are systematically larger than that from the analytical model, but within $2 \%$. This difference comes from the linearization of the temperature used in the analytical model, see Sections. 3.1.1, 3.1.2.

The comparison between dimensionless temperature profiles obtained from the continuum and kinetic models is divided into two categories according to the temperature ratio $\mathcal{T}$. 

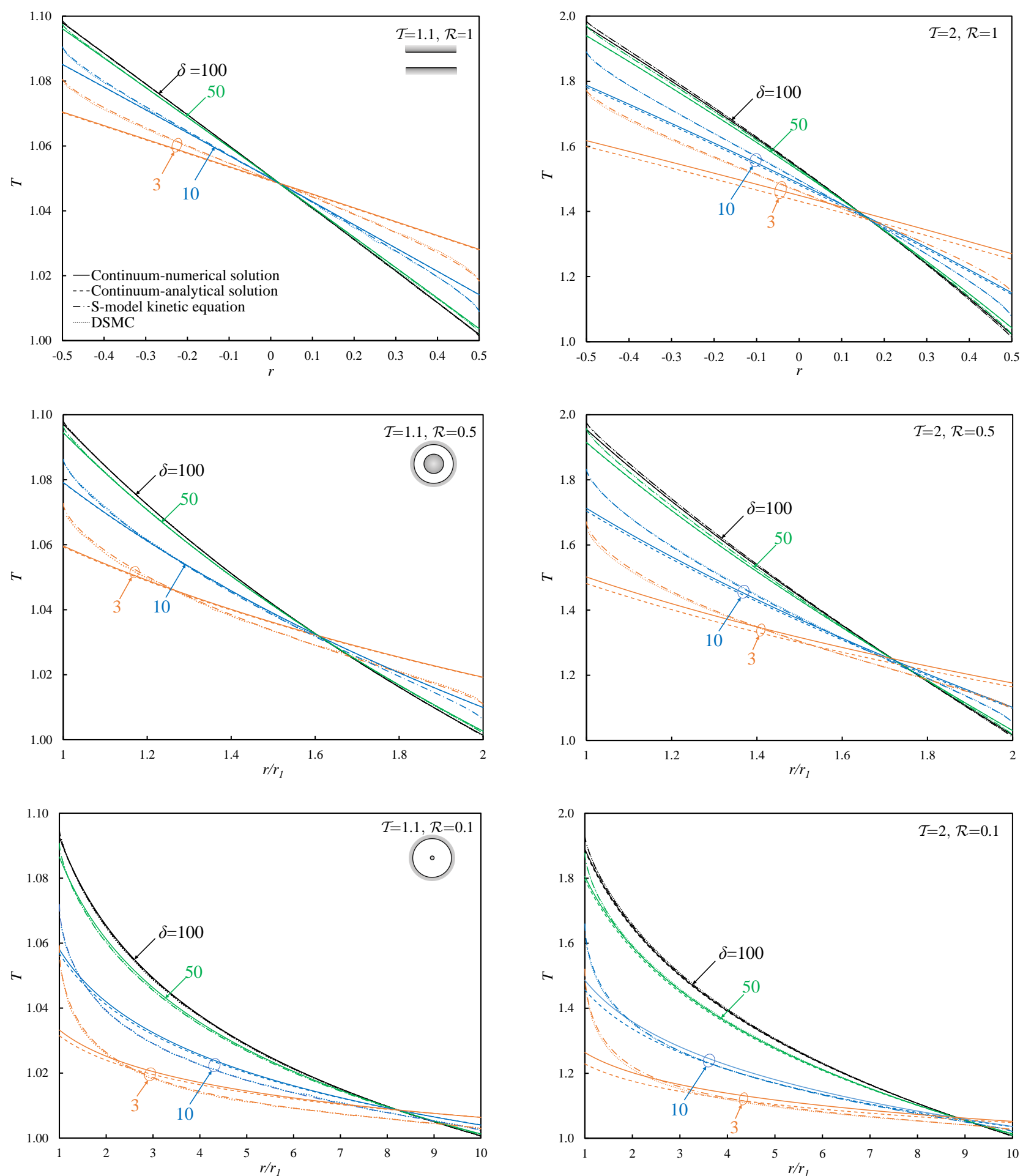

Fig. 2. Dimensionless temperature profiles between plates and cylinders for all combination of $\mathcal{R}$ and $\mathcal{T}$ and different values of rarefaction parameters $\delta=100,50,10$ and 3 in case $\alpha=1$.

- For $\mathcal{T}=1.1$ and all $\mathcal{R}$, there is a good agreement between the two types of models for $\delta=100$ and 50 . For $\delta=10$, the agreement is good in the core of the gap (away from the walls), except for the case $\mathcal{R}=0.1$. Deviations are observed close to the walls. For smaller $\delta$, there is disagreement even within the core of the gap for all aspect ratios $\mathcal{R}$.

- For $\mathcal{T}=2$, the agreement between the continuum and kinetic models for $\delta=100$ and 50 is not as close as it is for $\mathcal{T}=1.1$. However, for smaller $\delta \leq 10$, the disagreement increases as $\delta$ decreases. 

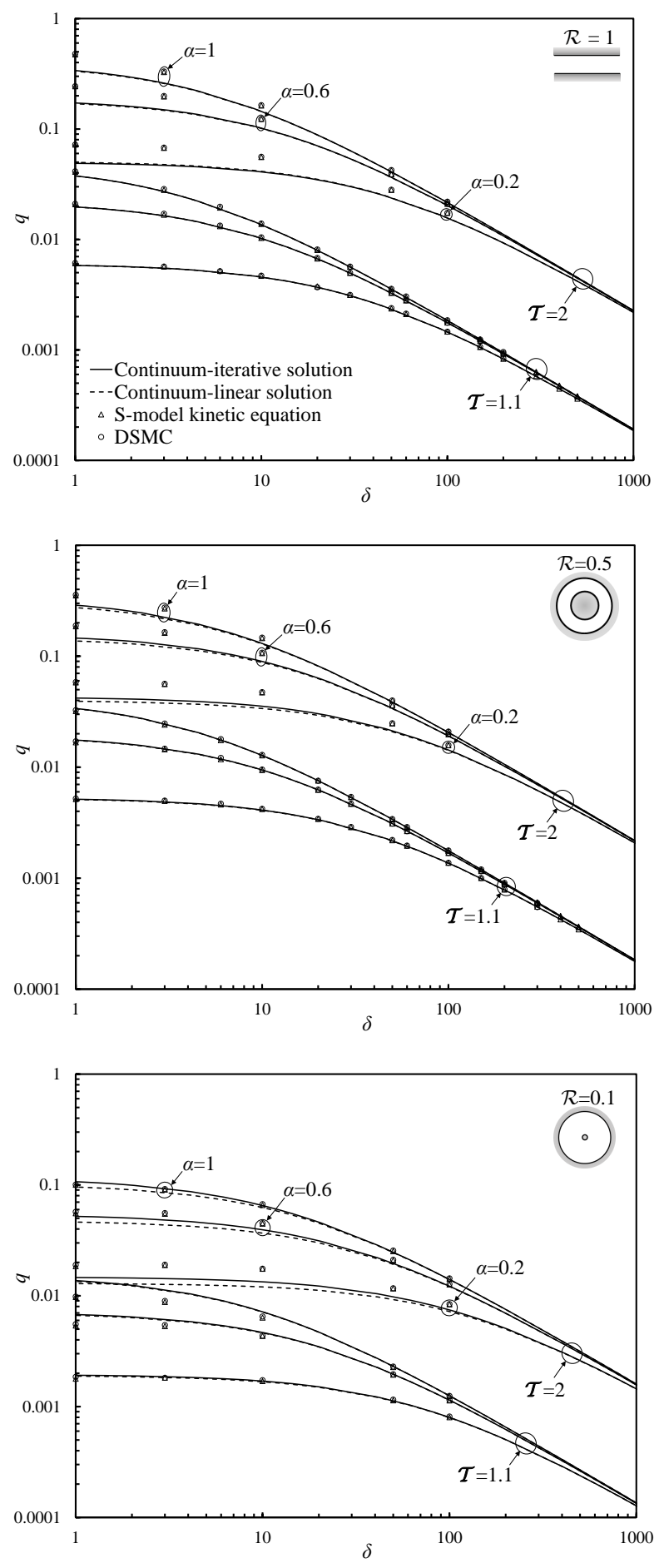

Fig. 3. Dimensionless heat flux as function of the rarefaction parameter $\delta$ obtained for all combination of $\mathcal{R}$ and $\mathcal{T}$ and different values of thermal accommodation coefficient $\alpha$.

Figure 2 visibly shows that the temperature-jump at the walls increases as $\delta$ decreases. The temperature-jump at the hotter wall is larger than the corresponding one at the cooler wall and it increases as $\mathcal{R}$ decreases. Furthermore, as $\mathcal{T}$ increases, the temperature-jump at both walls increases. However, the increase of $\mathcal{T}$ has a larger effect on the jump at the inner wall than the outer wall. This is because the gas becomes more rarefied as its temperature increases.

Overall, the temperature-jumps at the walls predicted by the continuum models increase as $\delta$ and $\mathcal{R}$ decrease, and $\mathcal{T}$ increases and they are larger than those predicted by the kinetic models. 


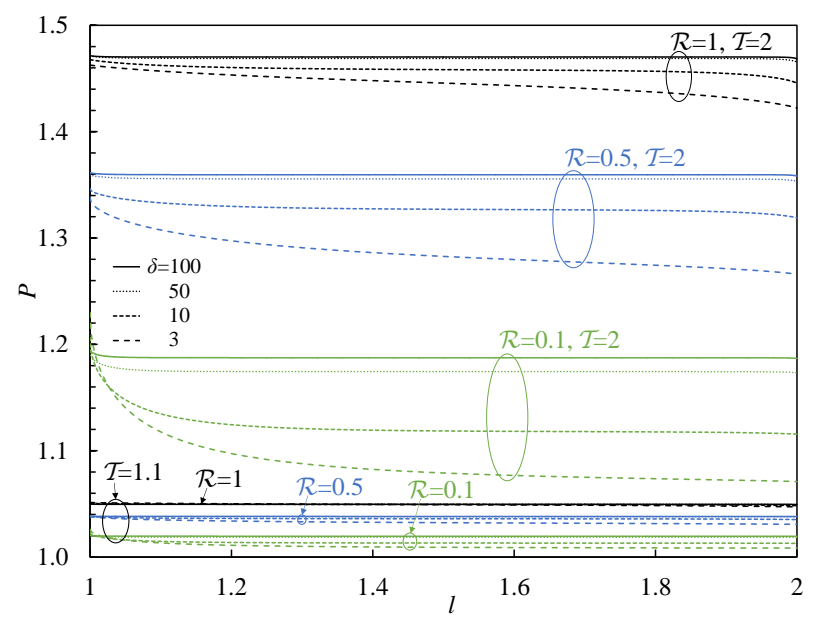

Fig. 4. Dimensionless pressure profiles between plates and cylinders for all combination of $\mathcal{R}$ and $\mathcal{T}$ and different values of rarefaction parameters $\delta=100,50,10$ and 3 in case $\alpha=1$. Black lines are for parallel plates $(\mathcal{R}=1)$, blue and green lines are for coaxial cylinders with $\mathcal{R}=0.5$ and $\mathcal{R}=0.1$, respectively. $l=r$ for $\mathcal{R}=0.5$ and $l=1-R_{1}+r$ for $\mathcal{R}=1$ and 0.1 .
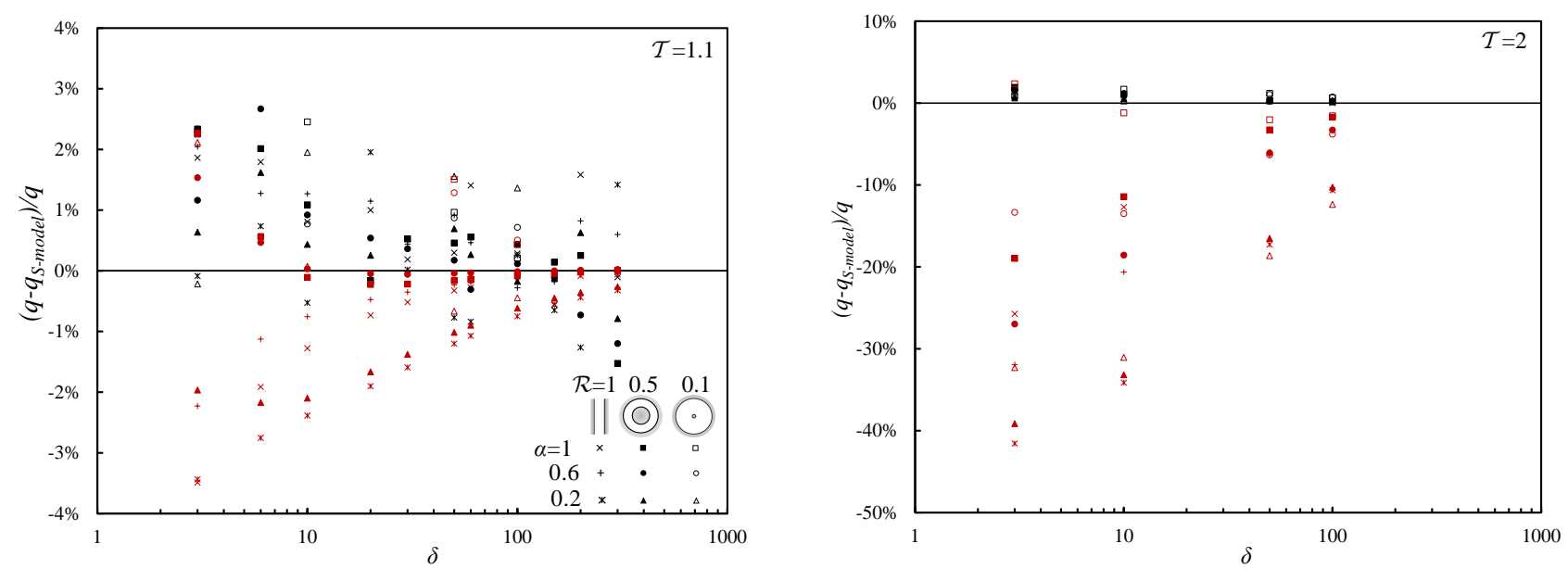

Fig. 5. Percent deference of the dimensionless heat flux $q$ between S-model and DSMC (black), and Numerical-continuum (red) models as function of the rarefaction parameter $\delta$ obtained for all combinations of $\mathcal{T}$ and $\mathcal{R}$ and different values of thermal accommodation coefficient $\alpha$.

\subsection{Dimensionless heat flux}

Figure 3 shows the dimensionless heat flux $q$, which is the quantity of practical interest in this paper, plotted as a function of rarefaction parameter $\delta$ for all combinations of $\mathcal{T}$ and $\mathcal{R}$ considered in this paper and for $\alpha=1,0.6$ and 0.2 with $\alpha=\alpha_{1}=\alpha_{2}$. Results from kinetic (S-model and DSMC) and continuum (numerical and analytical) approaches are included.

It is clear from this figure that the value of $q$ increases as $\delta$ decreases and $\alpha$ and $\mathcal{T}$ increase. The values of $q$ obtained from both continuum models (numerical and analytical) are in a good agreement for case $\mathcal{T}=1.1$, regardless $\mathcal{R}$ and $\alpha$. For $\mathcal{T}=2$, the discrepancy between the two models increases as $\delta, \alpha$, and $\mathcal{R}$ decrease. The value of $q$ for the continuum numerical model is systematically larger than the continuum-analytical model.

At $\mathcal{T}=1.1$, the continuum and kinetic results are in good agreement for all values of $\delta$, except for cases $\mathcal{R}=0.1$ and $\alpha=1$. The reason of this discrepancy is not clear and may be investigated further. For $\mathcal{T}=2$, the agreement is good for $\mathcal{R}=0.1$ and $\alpha=1$, but is less good as $\alpha$ decreases and $\mathcal{R}$ increases.

The disagreement between the continuum and kinetic models, especially at large $\mathcal{T}$, may be explained from the profiles of dimensionless pressure along the $r$-axis. Figure 4 shows these profiles between the cylinders and plates obtained from the S-model kinetic equation in the case of full accommodation of the molecules at the walls. The results are plotted for $\mathcal{T}=1.1$ and $2, \mathcal{R}=1,0.5$, and 0.1 , and $\delta=100,50,10$, and 3 . The $r$-axis was scaled to be between 1 and 2 as described in the 
caption for convenience of comparison. From this figure, it can be seen that, in all cases, the pressure varies along the $r$-axis between the cylinders and plates and this variation is larger as $\delta$ and $\mathcal{R}$ decrease, and $\mathcal{T}$ increases. As mentioned earlier, the continuum models assume that the pressure is constant along the $r$-axis. Figure 4 shows that the pressure variation is very small for $\mathcal{T}=1.1$, which explains the good agreement obtained between the continuum and kinetic approaches. However, for $\mathcal{T}=2$, the pressure variation is significant for $\delta \leq 10$, therefore, the assumption of the constant pressure is not valid. This may explain the disagreement obtained in these cases.

Figure 5 gives the percentage difference of dimensionless heat flux $q$ between DSMC and S-model (shown in black symbols) and the continuum-numerical and S-model (shown in red symbols) for both temperature and aspect ratios, and for three values of the thermal accommodation coefficient considered in this work. It can be seen from this figure that the heat fluxes obtained from the DSMC and S-model are within 3\% of each other in all configurations. This difference decreases as $\delta$ increases and the gas approaches the continuum regime. It is also clear from Fig. 5 that, for the case $\mathcal{T}=1.1$, the percentage difference between the continuum-numerical and S-model is smaller than $4 \%$ for all values of $\mathcal{R}$, $\alpha$, and $\delta$, which is of the order of the difference between the DSMC and S-model approaches. However, in case $\mathcal{T}=2$, the percentage difference between the continuum-numerical and S-models is significantly larger than the corresponding difference between DSMC and S-model and of the order of $50 \%$ at small $\delta$. This quantitative comparison between the continuum and kinetic approaches clearly demonstrates that the continuum models are appropriate for simulating heat transfer for small temperature ratio, however, for large ratio their ability is largely reduced even at large value of $\delta \geq 50$.

In general, it can be concluded that the continuum models are accurate for $\delta \geq 10$ and $\mathcal{T}=1.1$, and for $\delta \geq 50$ and $\mathcal{T}=2$, regardless the aspect ratio.

\section{Conclusion}

The steady state heat transfer through rarefied gas confined between coaxial cylinders and parallel plates is studied using continuum and kinetic approaches in the slip regime. The calculations were conducted for different temperature and radius ratios. Both walls were assumed to have the same value of thermal accommodation coefficient $\alpha$.

The dimensionless temperature and heat flux were obtained from S-model and DSMC were compared to continuum numerical and analytical solutions of the Fourier equation subjected to the Lin \& Willis temperature-jump boundary condition at the walls.

The two continuum models were in good agreement for all values of $\delta$ and $\alpha$, with maximum difference less than $2 \%$, observed for case $\mathcal{T}=2$ and $\mathcal{R}=0.1$. The comparison between the continuum and kinetic approaches showed that the continuum models are valid in the slip regime for a temperature ratio of $\mathcal{T}=1.1$, however, for $\mathcal{T}=2$, they are only valid for $\delta \geq 50$.

It should be noticed that here the temperature boundary conditions are employed in the paper. However sometimes in practice it is important to be able to employ constant heat flux boundary conditions so that the heat exchange between the system and the surroundings can be controlled. The examples of the implementation of the heat flux boundary conditions are given in [26], [27] for the DSMC and kinetic approaches, respectively.

\section{Acknowledgments}

This work was supported by the US Department of Energy Office of Nuclear Energy University Program. This work was granted access to the HPC resources of Aix-Marseille Université, under the project number 13b015, financed by the project Equip@Meso (ANR-10-EQPX-29-01) of the program "Investissements d'Avenir" supervised by the Agence Nationale pour la Recherche.

\section{References}

[1] U.S. Dept. of Energy, Office of Civilian Radioactive Waste Management (OCRWM), 1987. "Doe/rw-0184, december.". Characteristics of Spent Nuclear Fuel, High-Level Waste, and Other Radioactive Wastes Which May Require Long-Term Isolation.

[2] Saling, J. H., and Fentiman, A., 2002. Radioactive Waste Management, 2 ed. Taylor and Francis, New York.

[3] Colmont, D., and Roblin, P., 2008. "Improved thermal modeling of snf shipping cask drying process using analytical and statistical approaches". Packaging, Transport, Storage \& Security of Radioactive Material, 19(3), pp. 160-164.

[4] Commission, U. N. R., et al., 2003. "Cladding considerations for the transportation and storage of spent fuel". ISG-11, Rev, 3.

[5] Kennard, E. H., 1938. Kitetic theory of gases. McCraw-Hill Book Company, Inc., New York.

[6] Shakhov, E. M., 1968. "Generalization of the Krook kinetic relaxation equation”. Fluid Dyn., 3(5), pp. 95-96.

[7] Graur, I. A., and Polikarpov, A., 2009. "Comparison of different kinetic models for the heat transfer problem". Heat and Mass Transfer, 46, pp. 237-244. 
[8] Graur, I., Ho, M. T., and Wuest, M., 2013. "Simulation of the transient heat transfer between two coaxial cylinders". Journal of Vacuum Science \& Technology A: Vacuum, Surfaces, and Films, 31(6), pp. 061603.1-9.

[9] Bird, G. A., 1994. Molecular Gas Dynamics and the Direct Simulation of Gas Flows. Oxford Science Publications, Oxford University Press Inc., New York.

[10] Cercignani, C., 1990. Mathematical methods in kinetic theory. Premuim Press, New York, London.

[11] Kogan, M. N., 1969. Rarefied gas dynamics. Plenum Press New York.

[12] Lin, J. T., and Willis, D. R., 1972. "Kinetic theory analysis of temperature jump in a polyatomic gas". Phys. Fluids, $15(1)$.

[13] Morse, T. F., 1964. "Kinetic model for gases with internal degrees of freedom". Physics of Fluids.

[14] Holway, L. H., 1966. "New statistical models in kinetic theory: methods of construction". Physics of fluids, 9(9), pp. 1658-1673.

[15] Welander, P., 1954. "On the temperature jump in a rarefied gas”. Ark. Fys., 7(5), pp. 507-553.

[16] Graur, I., and Ho, M. T., 2014. "Rarefied gas flow through a long rectangular channel of variable cross section". Vacuum, 101, pp. 328-332.

[17] Sone, Y., and Sugimoto, H., 1995. "Evaporation of a rarefied gas from a cylindrical condensed phase into a vacuum". Phys. Fluids, 7, p. 2072.

[18] Larina, I. N., and Rykov, V. A., 1998. "A numerical method for calculationg axisymmetric rarefied gas flows". Comput. Math. Math. Phys., 38(8), pp. 1335-1346.

[19] Shakhov, E. M., and Titarev, V. A., 2009. "Numerical study of the generalized cylindrical couette flow of rarefied gas". European Journal of Mechanics B/Fluids, 28, pp. 152-169.

[20] Hsu, S. K., and Morse, T. F., 1972. "Kinetic theory of parallel plate heat transfer in a polyatomic gas". Phys. of Fluids, 15(4), pp. 584-591.

[21] Stefanov, S., Gospodinov, P., and Cercignani, C., 1998. "Monte carlo simulation and navier-stokes finite differnce solution of rarefied gas flow problems". Physics of Fluids, 10(1), pp. 289-300.

[22] Gallis, M. A., Torczynski, J. R., Rader, D. J., and Bird, G. A., 2009. "Convergence behavior of a new dsmc algorithm". J. Comput. Phys., 228, pp. 4532-4548.

[23] Stefanov, S. K., 2011. "On DSMC calculations of rarefied gas flows with small number of particles in cells". SIAM J. Sci. Comp., 33(2), pp. 677-702.

[24] Hadjiconstantinou, N. G., Garcia, A. L., Bazant, M. Z., and He, G., 2003. "Statistical error in particle simulations of hydrodynamic phenomena”. J Comput Phys, 187(1), pp. 274-297.

[25] Schaaf, S. A., and Chambre, P. L., 1958. "Flow of rarefied gases". In Fundamental of Gasdynamics, H. W. Edmmons, ed., Vol. III. Princeton University Press, Princeton, pp. 687-739.

[26] Akhlaghi, H., Roohi, E., and Stefanov, S., 2012. "A newiterative wall heat flux specifying technique in dsmc for heating/cooling simulations of mems/nems". Int. J. Therm. Sci, 59, pp. 111-125.

[27] Meng, J., Zhang, Y., and M, R. J., 2015. "Numerical simulation of rarefied gas flows with specified heat flux boundary conditions". Commun. Comput. Phys., 17(5), pp. 1185-1200. 